\title{
CONDUCTION OF HEAT IN A FLAME OF ATOMIC HYDROGEN
}

\author{
by E. F. M. VAN DER HELD and H. J. VAN DEN BOLD
}

Physisch Laboratorium der Rijks-Universiteit te Utrecht

\section{Summary}

The transport of heat in a vertical hydrogen flame, in which the molecules are dissociated by an electric arc, has been calculated for the following cases: 1. Fluctuating heat production concentrated in one point.

2. Fluctuating heat production concentrated in one line of length 1 .

The calculation shows, that the fluctuation can not penetrate to the spots, where the temperature was measured. The temperatures along the axis of the flame, reduced to an appropriate scale, have been plotted against $1 / z$ and against $1 / \sqrt{ } z$ ( $z$ being the height above the heat source), in one of which cases a straight line should be obtained according to theory. The horizontal temperature distribution in the above mentioned scale is in good agreement with theory. Deviations are discussed and a table of theoretical values of temperature is given.

Temperatures have been measured by line reversal in the manner described in a paper by v. d. B o l d and $S \mathrm{~m} \mathrm{it}^{\mathbf{1}}$ ) and the results obtained have been collected in a table.

In a preceding paper ${ }^{1}$ ) a flame of atomic hydrogen and measurements of the temperature in this flame have been described. In this paper we will try to give a mathematical description of the observed phenomena, especially to get information about the fluctuation of the temperature in consequence of the motion of the arc. Stroboscopic observations have shown, that after the ignition the arc is carried along with the gas stream till in consequence of the increasing length the electric resistance has become so high, that the tension is risen to a voltage high enough to light a new shorter arc. Then the old arc extinguishes and the phenomenon repeats itself with the new arc. The time between two ignitions is about $10^{-3} \mathrm{sec}$.

In the jet beneath the arc the cold gas has a velocity of $300 \mathrm{~cm} / \mathrm{sec}$. and above the arc of $1200 \mathrm{~cm} / \mathrm{sec}$. In the following calculations we 
assume, that the velocity will be the same at different heights above the arc, the measurements giving no evidence that the hydrogen flame differs from an ordinary flame in this respect. The following considerations support this assumption.

The cause of the acceleration from 300 to $1200 \mathrm{~cm} / \mathrm{sec}$ can be:

1. The hot gases lighter than the surrounding air rise with increasing velocity;

2. The expansion of the arc gas raises the gas above it;

3. The expanding arc gas collides with the jet gas and is driven back by reaction;

The first mentioned cause accelerates the gas only from $300 \mathrm{~cm} / \mathrm{sec}$ to $310 \mathrm{~cm} / \mathrm{sec}$ at $3 \mathrm{~cm}$ and to $320 \mathrm{~cm} / \mathrm{sec}$ at $6 \mathrm{~cm}$ above the arc. According to the second cause the arc gas expands by electric heating. The velocity $v$ at a distance $r$ from the arc centre is given by the formula:

a. The arc regarded as a point source:

and

$$
v_{1}=\frac{q_{1}}{4 \pi r^{2} c_{p} \varrho_{0} T_{0}}
$$

$b$. as a line source:

$$
v_{2}=\frac{q_{2}}{2 \pi r c_{p} \varrho_{0} T_{0}} .
$$

The total heat production per second is $q_{1}={ }^{\prime} 360^{\circ} \mathrm{cal} / \mathrm{sec}$ (maximum).

Unpublished measurements by $\mathrm{M}$ e e $\mathrm{u}$ w s e $\mathrm{n}$ show, that at a distance of the electrodes of $2 \mathrm{~mm}$ the length of the arc is on the average $2 \mathrm{~cm}$, so that the heat production per second per $\mathrm{cm}$ amounts to $q_{2}=360 / 2=180 \mathrm{cal} / \mathrm{sec}, \mathrm{cm}$ (maximum).

The specific heat $c_{p}=3,40 \mathrm{cal} / \mathrm{gram},{ }^{\circ} \mathrm{C}$ (see further on). The product of specific density and absolute temperature $\varrho T=\varrho_{0} T_{0}=$ $=2,45 \cdot 10^{-2}$ gram ${ }^{\circ} \mathrm{K} / \mathrm{cm}^{3}$.

Then in case $a: v_{1}=340 / r^{2} \mathrm{~cm} / \mathrm{sec}$ and in case $b: v_{2}=340 / r$ $\mathrm{cm} / \mathrm{sec}$. At short distances $(r<1 \mathrm{~cm})$ the arc will behave as a line source (case $b$ ) and at greater distances as a point source (case $a$ ).

According to the formulae $v$ will be $37.7 \mathrm{~cm} / \mathrm{sec}$ at $3 \mathrm{~cm}$ above the arc and $9,4 \mathrm{~cm} / \mathrm{sec}$ at $6 \mathrm{~cm}$. This does not fit the facts. In reality the acceleration is much larger in the upward than in the lateral direction. 
Third cause: The gas tries to expand in the same way in all directions. In the downward direction the expanding gas encounters the jet gas. In the jet the pressure will rise till the jet stream overcomes the obstacle and the expanding gas is driven back with its own velocity, given by the formula $a$ or $b$, at the boundary, increased by the velocity of the jet gas: $v_{1}-v_{0}=900 \mathrm{~cm} / \mathrm{sec}=340 / r^{2}$. (3-dimensional: $a$ ) or 340/r (2-dimensional: $b$ ). In accordance here with, we must have, for the distance $d$ of the arc above the boundary between the arcand jet gases: $0.38<d<0.62 \mathrm{~cm}$. Moreover, this distance must be smaller than the smallest distance between the arc and the opening of the tube, which distance is larger than $1 \mathrm{~cm}$. Only the third cause can therefore give an explanation of the considerable increase of velocity. The gas once having a velocity of $1200 \mathrm{~cm} / \mathrm{sec}$ there is no reason why the velocity should change any more. This reasoning is supported by the fact, that the flame contour is cylindrical.

As the arc can move in the hot gas, it will find the shortest path between the electrodes through the hot gas. This path lies as near as possible to the boundary between hot and cold gases on the hot side, so that boundary and arc move with the same constant velocity, only a little smaller than the maximum velocity, of the cold gas, being twice the mean velocity in the tube. Measurements by $\mathrm{M}$ e e $\mathrm{u}$ w s e $\mathrm{n}$ on the length of the arc as a function of time, show, that the velocity of the top of the arc is nearly equal to the mean velocity of the cold gas in the tube.

A mathematical description of the phenomenon should give:

1e. the distribution of the gas velocity in the flame;

2e. the distribution of the temperature;

$3 \mathrm{e}$. the periodic movement of the arc.

The simultaneous solution of the differential equations for velocity and temperature being too difficult, we have assumed, that the velocity is constant throughout the flame, and we have substituted the moving arc by a fixed horizontal one with varying heat production. The unmodified differential equation of Fourier reads:

$$
\frac{\partial}{\partial x}\left(\lambda \frac{\partial \theta}{\partial x}\right)+\frac{\partial}{\partial y}\left(\lambda \frac{\partial \theta}{\partial y}\right)+\frac{\partial}{\partial z}\left(\lambda \frac{\partial \theta}{\partial z}\right)=c_{p} \varrho\left(\frac{\partial \theta}{\partial t}+u \frac{\partial \theta}{\partial x}+v \frac{\partial \theta}{\partial \lambda}+w \frac{\partial \theta}{\partial z}\right)
$$
with:

$\theta=$ rise of temperature;

$\lambda=$ thermal conductivity;

$$
\begin{aligned}
& c_{p}=\text { specific heat at constant } \\
& \text { pressure; }
\end{aligned}
$$


$z=$ height above the arc; $\quad \varrho=$ density;

$x=$ horizontal distance from the arc; $t=$ time;

$y=$ distance from the centre along the arc;

$w=$ velocity in the $z$-direction (constant); $u ; v=$ velocities in $x$ and $y$ direction $(=0)$;

$\lambda, c_{p}$ and $\varrho$ are functions of the temperature. In order, to simplify the equation of $\mathrm{F}$ ourier we put $\lambda d \theta=\lambda_{0} d \vartheta, \lambda_{0}$ being a constant, say the conductivity at $2200^{\circ} \mathrm{K}$, so that $\vartheta=1 / \lambda_{0} \int_{0}^{\theta} \lambda d \theta$.

Putting $\lambda / c_{p} \varrho=a$, the thermal diffusivity: we find $a \Delta \vartheta=\partial \vartheta / d t+$ $+w \partial \vartheta / \partial z$, the equation of $\mathrm{F}$ ou ri e r with constant coefficient of

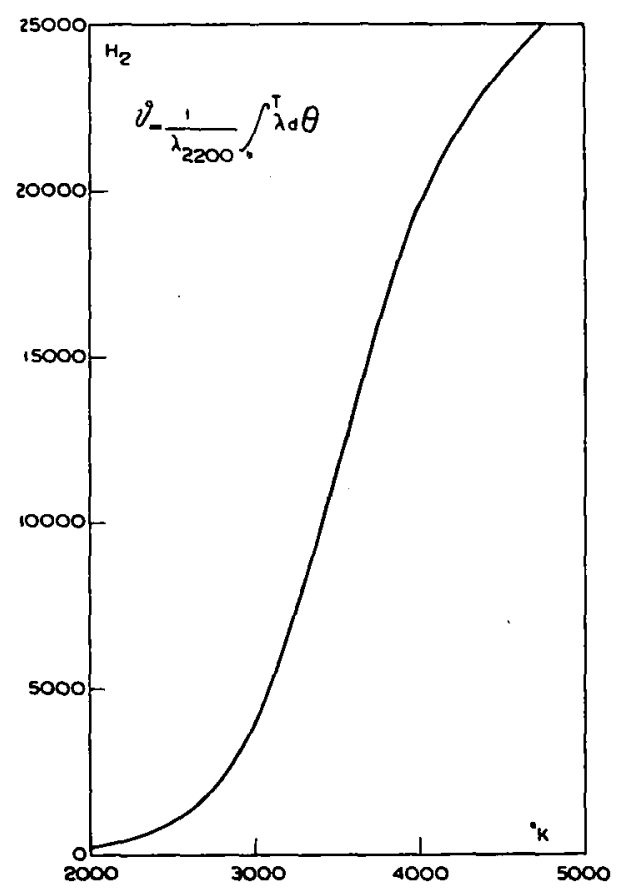

Fig. 1. Temperature function.

thermal conductivity, but in our case not with constant thermal diffusivity. We shall call $\vartheta$ the temperature function. The thermal conductivity as a function of the temperature is given in fig. $1^{2}$ ); the specific heat per volume unit in fig. $2^{3}$ ) and the thermal diffusivity in fig. 3. Only between 3000 and $3800^{\circ} \mathrm{K}$ the thermal diffusivity is nearly constant. Between $2200^{\circ} \mathrm{K}$, the temperature of the surrounding 
"cold" hydrogen, and $3000^{\circ} \mathrm{K}$ the thermal diffusivity increases from 25 to $200 \mathrm{~cm}^{2} / \mathrm{sec}$. As no solution of the equation of F o u rie r with variable thermal diffusivity is known to us we have set up our calculation with a constant value of this quantity $\left(a=200 \mathrm{~cm}^{2} / \mathrm{sec}\right)$ accepting, that the solution will be an approximation only. Yet the solution turns out to give the desired information for the damping of the oscillation of the temperature in the gas stream.

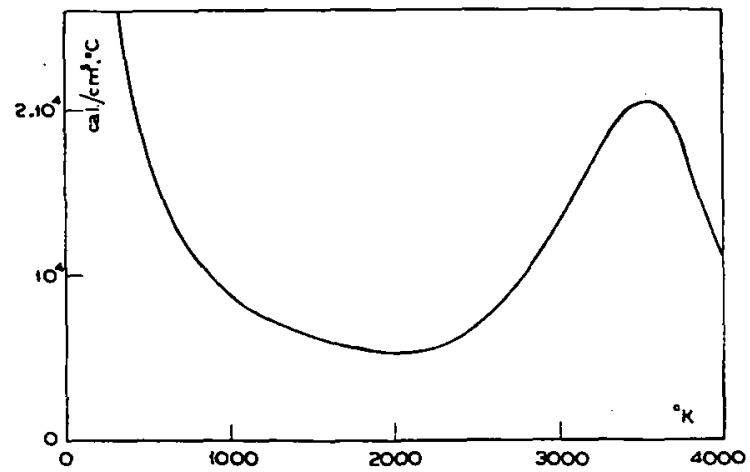

Fig. 2. $\mathrm{H}_{2}$ Specific heat per volume unit.

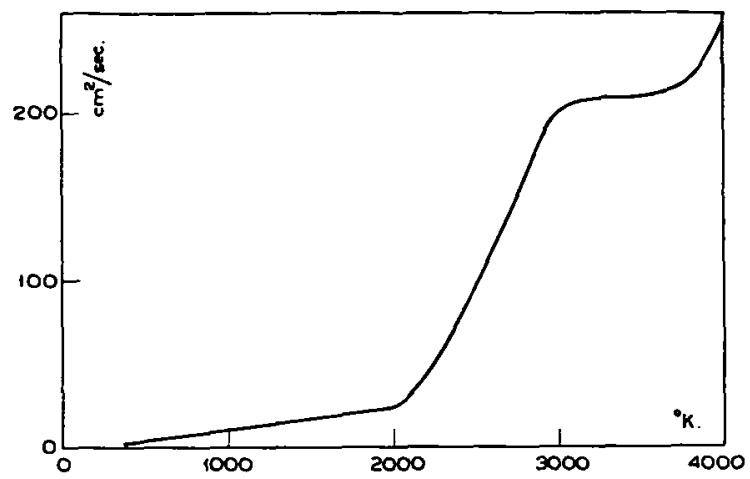

Fig. $\mathrm{H}_{2}$ Thermal diffusivity.

If a source of heat be present at the point $(0,0,0)$ in a flame, of which the velocity of gas flow is $W$ in the $z$-direction, this source of heat producing $q_{1}(1-\cos \omega t) \mathrm{cal} / \mathrm{sec}$, the temperature function in the point $(x, y, z)$ will have increased to:

$$
\vartheta_{1}=\frac{q_{1}}{8 \pi^{3 \cdot 2} \lambda_{0} \sqrt{ } a} \int_{-\infty}^{t} \frac{e^{-\frac{r^{2}}{4 \pi(t-\tau)}+\frac{W}{a} z-\frac{W^{2}(t-\tau)}{4 a}}}{(t-\tau)^{3 / 2}}(1-\cos \omega \tau) d \tau\left(r^{2}=x^{2}+y^{2}+z^{2}\right)
$$




$$
\vartheta_{1}=\frac{q_{1} e^{\frac{W z}{2 a}}}{4 \pi \lambda_{0} r}\left[e^{-\frac{w^{2}}{2 a}},-e^{-\frac{r}{2 a}} \sqrt{\frac{1 W^{2}+\sqrt{\frac{W_{4}}{4}+\omega^{2 a 2}}}{2}} \cos \left\{\omega t-\frac{r}{2 a} \sqrt{\left.\left.-\frac{1}{2} W^{2}+\sqrt{\frac{W^{4}}{4}+4 \omega^{2} a^{2}}\right\}\right]}\right.\right.
$$

If the production of heat is not limited to one point but is spread regularly over a straight line of length 1 perpendicular to the $z$-axis, $q_{1}$ must be substituted by $q_{2} d y_{0}$ and $y$ by $y-y_{0}$. Then the temperature function at the point $(x, y, z)$ is given by:

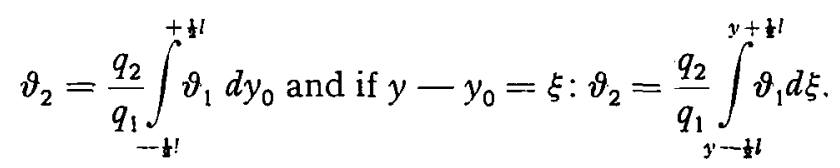

This would leave us with integrals of the form (putting $x^{2}+z^{2}=\varrho^{2}$ ):

$$
I=\int_{y-\frac{1}{t} l}^{y+t l} \frac{e^{-A d \sqrt{\rho^{2}+\xi^{2}}}}{\sqrt{\varrho^{2}+\overline{\xi^{2}}}} d \xi
$$

which can be trasformed into $\mathrm{S}$ o $\mathrm{m}$ mer feld integrals by substituting $i \varrho \sin \beta$ for $\xi$ ( $A$ can be real or complex, $\beta$ is complex and the real axis is intersected at $\beta=0$ ). The integral is therefore,

$$
I=i \int_{\arcsin i\left(\frac{y-\frac{1}{\rho}}{\rho}\right)}^{\arcsin i\left(\frac{y+t}{\rho}\right)} e^{-A \rho \cos \beta} d \beta
$$

As far as we know these integrals have never been put into numerical tables. If $y+\frac{1}{2} l$ is $\ll \varrho$, we find the approximating value:

$\sqrt{\frac{\pi}{2 \varrho A}} e^{-A \rho}\left\{\psi\left(\mid \sqrt{\left.A \sqrt{\varrho^{2}+\left(y+\frac{1}{2} l\right)^{2}}-A \varrho\right)}+\psi\left(\sqrt{\left.A \sqrt{\varrho^{2}+\left(y-\frac{1}{2} l\right)^{2}}-A \varrho\right)}\right\}\right.\right.$

with an error of $1 \%, \psi$ being the integral of $G$ a u s $\psi(u)=2 / \sqrt{ } \pi$ $\int_{0}^{z} e^{-t 2} d t$, and in the axis of the flame $(y-0): l / z e^{-A z}$ with a maximum error of $5 \%$. This means, that at a distance $\varrho \gg \frac{1}{2} l$ we may consider the arc as a 3-dimensional point source of heat, which case we have treated already. If $l \gg \varrho$, we may consider the source as one of infinite length and get:

$I=i \int_{-i \infty}^{+i \infty} e^{-A \rho \cos \beta} d \beta=i \pi H_{0}^{(1)}(i A \varrho)\left(H_{0}^{(1)}=\right.$ Hankel function $)$ 
so that:

$$
\vartheta_{2}=\frac{q_{2}}{4 \lambda} e^{\frac{W_{s}}{2 i}}\left\{i H_{0}^{(1)}\left(i \frac{W \varrho}{2 a}\right)-h \sin (\omega t+\eta)\right\}
$$

and the real numbers $h$ and $\eta$ can be found from the relation:

$$
h e^{i \eta}=H_{0}^{(1)}\left\{\frac{g}{2 a}\left(-\sqrt{-\frac{1}{2} W^{2}+\sqrt{\frac{W^{4}}{4}+4 \omega^{2} a^{2}}}+i \sqrt{\left.\frac{1}{2} W^{2}+\sqrt{\frac{W^{4}}{4}+4 \omega^{2} a^{2}}\right)}\right\}\right.
$$

We are not concerned with $\eta$, as it only affects the phase, while on the contrary $h$ is very important for the damping of the periodic fluctuation of the temperature.

In our points of measurement the first term of the semiconvergent series of Hankel differs $2 \%$ at the utmost from the Hankel function itself, so that

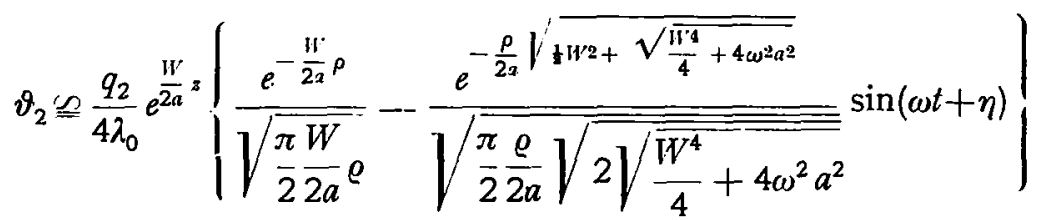

of foremost interest is the ratio between $h$ and

$$
\begin{gathered}
i H_{0}^{(1)}\left(i \frac{W}{2 a} \varrho\right), \\
\frac{h}{i H_{0}^{(1)}\left(\frac{W}{2 a} \varrho\right)}=\frac{e^{\frac{\rho}{2 a}\left\{W-\sqrt{1 W^{2}+\sqrt{\left.\frac{W^{4} 4}{4}+4 \omega^{2} a^{2}\right\}}}\right.}}{\left(1+\frac{16 m^{2} a^{2}}{W^{4}}\right)^{1 / s}}
\end{gathered}
$$

If the following quantities be taken from the experiments:

$$
\begin{array}{rlrl}
W & =1200 \mathrm{~cm} / \mathrm{sec} & x=0 \\
\omega & =8800 \mathrm{sec}^{-1} & z=3 \mathrm{~cm} \\
a & =200 \mathrm{~cm}^{2} / \mathrm{sec} &
\end{array}
$$

this ratio, which means the ratio between the periodic and the nonperiodic component the of temperature function, is $9,5 \cdot 10^{-4}$. The ratio between the two components of the temperature itself will be smaller than this number.

The preceding calculations apply to the two-dimensional case. In 
the three-dimensional (one point source) case the above-mentioned ratio will be:

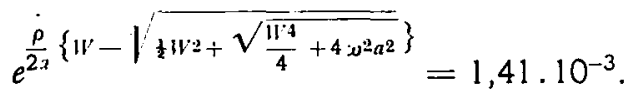

In both cases the fluctuation in the considered spot $(3 \mathrm{~cm}$ above the arc) can scarcely or not at all be observed.

One might object that the variation in the heat production $q_{1}$ $(1-\cos \omega t)$, as used in the calculation, will be far from the real one. We have therefore repeated the reckoning with an other function, with the same mean heat production, but with sharp and very high peaks:

$$
q_{1} \frac{2^{2 n}(n !)^{2}}{(2 n) !} \cos ^{2 n} \frac{\omega t}{2} .
$$

If $n$ is a very great number e.g. $>5000$, the first terms of the expanded series are: $q_{1}\{1+2 \cos \omega t+2 \cos 2 \omega t+2 \cos 3 \omega t+\ldots\}$.

The higher terms will damp out much quicker than the first term. So we have only to consider the second term, which is essentially the same as the second term of the first considered function. The proportion of the periodic and the non-periodic component will be $2,82.10^{-3}$ reckoning with the factor 2 of the terms of the peakfunction (3-dimensional case).

However poorly the chosen solution of the differential equation may describe the phenomenon, we can conclude, that in reality the fluctuation is fully damped out at the lowest spot used for observations.

Once having obtained our solutions for the distribution of the mean temperature, we have checked these solutions by our measurements to find out to what degree they can describe this distribution in our flame. For the value of the temperature function at the $z$-axis we find:

$$
\begin{array}{ll}
\vartheta_{2} \cong \frac{q_{2}}{2 \lambda_{0} \sqrt{\pi \frac{W z}{a}}} & \text { in the 2-dimensional case; } \\
\vartheta_{1} \cong \frac{q_{1}}{4 \pi \lambda_{0} z} & \text { in the 3-dimensional case; }
\end{array}
$$

If $\vartheta$ is plotted against $1 / \sqrt{ } z$ and $1 / z$ respectively, the result to be expected is in both cases a straight line, intersecting the $\vartheta$-axis in one 
single point, the value of the temperature function corresponding with the temperature of the flame when undisturbed by atomic hydrogen: the common hydrogen flame.

From the graphs (fig. 4 and 5) the conclusions can be drawn, that the two-dimensional solution describes the experimental outcome much better than the three-dimensional one. The temperature of the non-disturbed flame would in that case, be $2400^{\circ} \mathrm{K}$, while a $2200^{\circ} \mathrm{K}$ value has been assumed by v. d. B old and S m it. The slope of the lines is

$$
H=-\frac{q_{2}}{2 \lambda_{0} \sqrt{\pi \frac{W}{a}}} .
$$

The separate curves differ by different energy input.

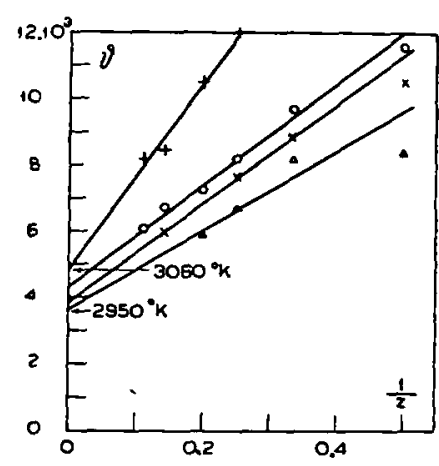

Fig. 4.

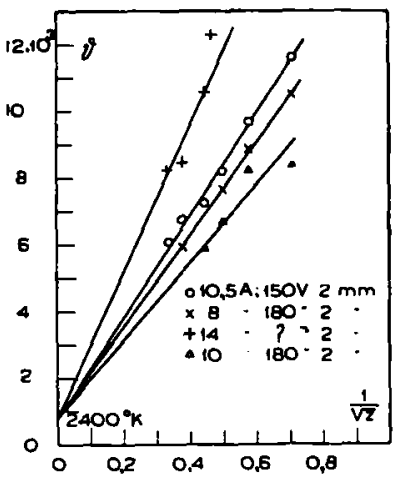

Fig. 5.

The incoming energy is partly radiated, principally by the electrodes. If $l$ is the mean length of the arc, the total heat production of the arc will be $q_{2} l$. This energy equals the electric input. We can ccrrect $q_{2} l$ for the radiation of the electrodes $(5 \%)$.

If we neglect the remaining radiation, of the arc itself, we are able to calculate $q_{2}$ for the lines of the $\odot$ and $\times$ marks.

For $\odot: H=1,52.10^{4}$ and $q_{2}=1,29.10^{10} \mathrm{erg} / \mathrm{sec}, \mathrm{cm}$ and for $\times$ : $H=1,38.10^{4}$ and $q_{2}=1,17.10^{10} \mathrm{erg} / \mathrm{sec} . \mathrm{cm}$.

For $\odot q_{2} l=1,51.10^{10} \mathrm{erg} / \mathrm{sec}$, so that $l=1,16 \mathrm{~cm}$, and for $\times q_{2} l$ $=1,37.10^{10} \mathrm{erg} / \mathrm{sec}$, so that $1=1.17 \mathrm{~cm}$. Unpublished measurements by P. I. M e e u w s e $\mathrm{n}$ carried out in this laboratory show, that the mean length of the arc, which has the form of a horseshoe, 
is in reality $4 \mathrm{~cm}$. Though the 2 -dimensional solution complies better with the experiments about the temperature fall along the axis of the flame, the formula of the slope does not agree with the energy input. Let us, therefore, consider the three-dimensional case.

Here the slope is for $\bigcirc: H=q_{1} / 4 \pi \lambda_{0}=1,54.10^{4}$ and $q_{1}=1,93.10^{10}$ $\mathrm{erg} / \mathrm{sec}$, that is $30 \%$ too high and for $\times: H=1,49.10^{4}$ or $q_{1}=1,87.10^{10} \mathrm{erg} / \mathrm{sec}, 37 \%$ too high. Though the experimental data cannot be described by straight lines intersecting in one single point of the $\vartheta$-axis in a $\vartheta, 1 / z$-diagram, the formula of the slope agrees in this case better with the energy input.

As a matter of fact we must conclude, that the method of representation in a $\vartheta, 1 / \sqrt{ } z$ diagram by chance gives straight lines intersecting the $\vartheta$-axis in one single point corresponding with the expected temperature.

The temperature in a cross-section can be calculated from the formula 2 and 3. Measurements have been made for $z=3$.

TABLE I

\begin{tabular}{|c|c|c|}
\hline \multicolumn{3}{|c|}{$Z=3 \mathrm{~cm}$} \\
\hline$\varrho$ & $\begin{array}{c}\theta_{1} \text { (3-dim.) } \\
{ }^{\circ} \mathrm{K}\end{array}$ & $\begin{array}{c}\theta_{\mathbf{Z}} \text { (2-dim.) } \\
{ }^{\circ} \mathrm{K}\end{array}$ \\
\hline 0.0 & 3541 & 3541 \\
0.1 & 3537 & 3537 \\
0.2 & 3526 & 3526 \\
0.3 & 3510 & 3510 \\
0.4 & 3487 & 3487 \\
0.5 & 3456 & 3458 \\
0.6 & 3422 & 3425 \\
0.7 & 3385 & 3390 \\
0.8 & 3343 & 3353 \\
0.9 & 3302 & 3313 \\
1.0 & 3255 & 3268 \\
1.1 & 3207 & 3220 \\
1.2 & 3157 & 3172 \\
1.3 & 3104 & 3125 \\
1.4 & 3051 & 3079 \\
1.5 & 3012 & 3034 \\
1.6 & 2970 & 2990 \\
1.7 & 2929 & 2947 \\
1.8 & 2888 & 2905 \\
\hline
\end{tabular}

The horizontal temperature distribution is calculated for an environment temperature of $2400^{\circ} \mathrm{K}$ and a value of the thermal diffusivity $a=200 \mathrm{~cm}^{2} / \mathrm{sec}$ in the 2 - and the 3-dimensional case (table I). 
The temperature in the centre has been adapted to the corresponding central temperature given by measurement since the correspondance between measurement and calculation is discussed already for the axis of the flame. The differences between the 2- and 3-dimensional solutions are so small in the region, where the measurements

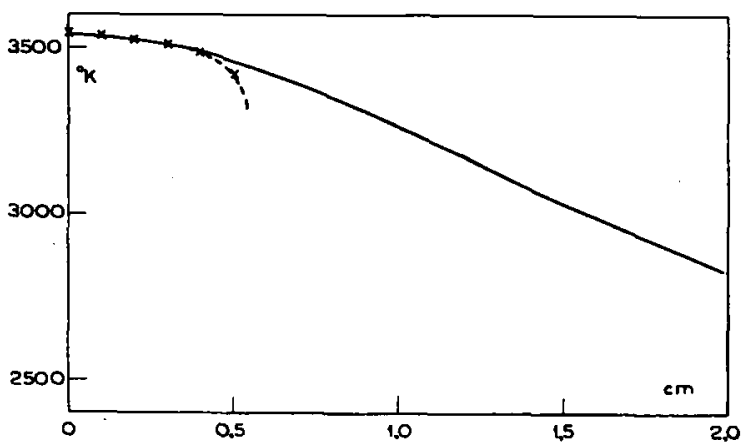

Fig. 6. Horizontal distribution of temperature calculated $\left(a=200 \mathrm{~cm}^{2} / \mathrm{sec}\right)$.

$x-x$ measurements of $\mathrm{V}$ a $\mathrm{n}$ d e $\mathrm{n}$ B old (hydrogen arc).

are reliable, that they can be entirely neglected. The calculated distribution is in good agreement with the measurements, the deviations at $5 \mathrm{~mm}$ arising from the inaccuracy of measurements at low intensities (50\% and more can be expected) (fig. 6).

Received November 23rd, 1948.

REFERENCES

1) H. J. van de n Bold and J. A. S m it, Physica 12, 475, 1946.

2) H. Brinkma n, Thesis Utrecht 1937, page 71 .

3) F. He n n ing, Wärmetechnische Richtwerte VDI-Verlag 1938, Berlin. 